\title{
The demand for a healthy diet: estimating the almost ideal demand system with infrequency of purchase
}

Article

Accepted Version

Tiffin, R. and Arnoult, M. (2010) The demand for a healthy diet: estimating the almost ideal demand system with infrequency of purchase. European Review of Agricultural Economics, 37 (4). pp. 501-521. ISSN 0165-1587 doi: https://doi.org/10.1093/erae/jbq038 Available at https://centaur.reading.ac.uk/16773/

It is advisable to refer to the publisher's version if you intend to cite from the work. See Guidance on citing.

To link to this article DOI: http://dx.doi.org/10.1093/erae/jbq038

Publisher: Oxford University Press

All outputs in CentAUR are protected by Intellectual Property Rights law, including copyright law. Copyright and IPR is retained by the creators or other copyright holders. Terms and conditions for use of this material are defined in the End User Agreement.

www.reading.ac.uk/centaur 
Central Archive at the University of Reading

Reading's research outputs online 


\title{
The demand for a healthy diet: estimating the almost ideal demand system with infrequency of purchase.
}

\author{
Richard Tiffin ${ }^{1}$ \\ Department of Food Economics and Marketing, University of Reading, UK \\ Matthieu Arnoult \\ Land Economy and Environment, Scottish Agricultural College, Edinburgh, UK.
}

\section{Summary}

A Bayesian method of estimating multivariate sample selection models is introduced and applied to the estimation of a demand system for food in the UK to account for censoring arising from infrequency of purchase. We show how it is possible to impose identifying restrictions on the sample selection equations and that, unlike a maximum likelihood framework, the imposition of adding up at both latent and observed levels is straightforward. Our results emphasise the role played by low incomes and socio-economic circumstances in leading to poor diets and also indicate that the presence of children in a household has a negative impact on dietary quality.

Keywords: Almost Ideal Demand System, Censoring, Bayesian Estimation, Diet and Health.

JEL classification: C11, C24, D12

\section{Introduction}

It is increasingly recognised that diet related chronic disease represents one of the most significant public health challenges of the twenty-first century. For example the prevalence of overweight and obesity has grown rapidly since the 1980s and, according to the Health Survey for England, in 2008 $66 \%$ of the adult males and $57 \%$ of adult females had a BMI greater than 25 while $25 \%$ of adult males and $24 \%$ of adult females were obese (BMI greater than 30). In addition to obesity, the roles that can be played by fruit and vegetables in the prevention of cancer also commands attention as do the impacts of dietary fat composition on fat and lipoprotein levels in the blood and associated impacts on heart disease. There is also a recognition (Drewnowski, 2004, Dowler, 2003) that diet related health problems are not evenly distributed in society. The first objective of this paper is therefore to apply an economic framework to analyse the factors which contribute to this uneven distribution. To do this we estimate a demand model which incorporates socio-demographic variables using micro-data.

Micro-data are in general subject to the econometric problem of censoring. In demand analysis this arises because most households do not purchase all of the commodities available to them. Wales and Woodland (1983) introduce two econometric models for censored demand systems. They refer to the first model as the Kuhn-Tucker approach. As its name implies, it is based on the Kuhn-Tucker conditions for the consumer's optimisation problem. The econometric model is developed by adding a stochastic term to the utility function and as a result to the Kuhn-Tucker conditions. The conditions hold as an equality when an interior solution results and as an inequality when there is a corner solution. As a result the likelihood function is of a mixed discrete-continuous form (Pudney, 1989, p163) and is difficult to maximise for all but relatively small demand systems because of the numerical integration that is required in its evaluation. The intractability of the likelihood function has led to very few examples of the empirical implementation of the KuhnTucker approach; one example is Phaneuf et al. (2000). By contrast, the second model proposed by Wales and Woodland (1983), which they refer to as the Amemiya-Tobin approach, has been more

\footnotetext{
${ }^{1}$ Address for correspondence: Professor Richard Tiffin, Department of Food Economics and Marketing, University of Reading, PO Box 237, Reading, RG6 6AR UK. E-mail: j.r.tiffin@ reading.ac.uk.
} 
widespread in the literature. This second strategy for handling censoring is an application of the Tobit model (Tobin, 1958) as extended by Amemiya (1974) to the estimation of a system of equations. In this approach the demand model is derived without explicitly incorporating the nonnegativity conditions. Instead these are added to the estimated model by truncating the distribution of the stochastic demand choices to allow for a discrete probability mass at zero.

A number of strategies have been adopted to the estimation of the Amemiya-Tobin model. The direct estimation of the system by maximum likelihood has been problematic for reasons of computational complexity. Earlier attempts at the estimation of the Amemiya-Tobin model are therefore based on the two stage approach proposed by Heien and Wessells (1990) and developed by Shonkwiler and Yen (1999), which is itself an application of the Heckman (1979) method. The two step approach can be considered a generalisation of the Amemiya-Tobin approach because it comprises two sets of equations: in addition to the censored equations, additional equations are used to model the censoring and this allows the possibility of a difference between the models which determine the censoring rule and the continuous observations. The generalisation of the Tobit model in this way is discussed in the context of demand for a single good by Blundell and Meghir (1987) who refer to the model in which the sample selection rule and the continuous variable models differ as the double hurdle model, a model introduced originally by Cragg (1971). The double hurdle model is adapted by Blundell and Meghir (1987) to form an infrequency of purchase model which addresses the fact that within a truncated survey period, observed purchases may differ from actual demand as stocks are either built up or run down. Yen et al. (2003) note that two step estimation is consistent but inefficient and they return to maximum likelihood estimation of the original Amemiya-Tobin model using simulated and quasi maximum likelihood methods. These methods are generalised in Stewart and Yen (2004) and Yen (2005) in an analogous way to the generalisation offered by the two step estimators referred to above to account for differences in the processes determining selection and the continuous variable. They recognise that this generalisation is the multivariate equivalent of that proposed by Cragg (1971). Their models are estimated by maximum likelihood and are thus efficient.

The second objective of this paper is to contribute to this literature by applying Bayesian methods to the estimation of multivariate sample selection models. We also extend the range of models that have been estimated by maximum likelihood hitherto to the infrequency of purchase model and we incorporate the Wales and Woodland (1983, p. 273) approach to the imposition of adding-up which, as Pudney $(1989$, p157) notes, has been problematic in a maximum likelihood context.

\section{Infrequency of Purchase in the AIDS model}

The Infrequency of Purchase Model (IPM) is developed in order to accommodate the fact that censoring occurs in the demand system because a particular good may not be purchased by a household during the time that it is surveyed as it is consuming from stocks purchased in other time periods. Our approach draws on Blundell and Meghir (1987) in order to adapt the Almost Ideal Demand System (AIDS) to incorporate infrequency of purchase. The censoring rule that relates latent consumption $\left(q_{i h}^{*}\right)^{2}$ of the $i^{\text {th }}$ commodity by the $h^{\text {th }}$ household to observed purchases $q_{i h}$ is as follows:

$$
q_{i h}=\left\{\begin{array}{ll}
\frac{q_{i h}^{*}}{\Phi_{i t}} & y_{i h}=1 \\
0 & y_{i h}=0
\end{array},\right.
$$

\footnotetext{
${ }^{2}$ In this specification of the model we assume that latent consumption for all goods is non-zero. In other words we assume that all censoring is the result of infrequency of purchase. Whilst allowing for both infrequency of purchase and true corner solutions would be preferable, such an approach introduces an identification problem since the source of a zero may be either a non-purchase, a corner solution or both.
} 
where $\Phi_{i h}$ is the probability that a purchase is made $\quad p \quad y_{i h}=1$ and $y_{i h}$ is a binary variable which takes the value of 1 when a purchase occurs. The censoring rule (1) implies that there are two aspects to the latency of $q_{i h}^{*}$ according to whether $q_{i h}$ is observed or not. In cases where a purchase is made, latent consumption is related to observed purchases as follows:

$$
q_{i h}^{*}=q_{i h} \Phi_{i h} \quad \forall i \in C
$$

where:

$$
C=i: y_{i h}=1 \text {. }
$$

The latency in the observations where $q_{i h}$ is observed is addressed in the AIDS by defining the consumption shares for observations where a purchase is observed as follows:

$$
s_{i h}=\frac{p_{i h} q_{i h}^{*}}{\sum_{i \in C} p_{i h} q_{i h}^{*}} \quad \forall i \in C
$$

where $p_{i h}$ is the price of the $i^{\text {th }}$ good to the $h^{\text {th }}$ household and $q_{i h}^{*}$ is defined in equation (2).

In cases where no purchase is made $q_{i h}^{*}$ is non-zero as the good in question is consumed from stocks. In this case latent consumption cannot be computed using equation (2) because $q_{i h}$ is itself unobserved. Instead a data augmentation algorithm, which we discuss in section 3 , is used to replace the observed zeros with estimated values for latent consumption. The shares computed using equation (4) for observations where a purchase is observed sum to one by construction and therefore once they are combined with the latent shares corresponding to observations where no purchase is made, the adding up restriction will be violated. Wales and Woodland (1983, p270) show how this problem can be addressed in a maximum likelihood context. In order to do this an additional source of latency is introduced into the model for the shares, defined in equation (4), where a purchase is made. The effect of this is to adjust the shares defined in equation (4) to ensure that the combined latent shares for goods where a purchase is made and those where one is not satisfy the adding up restriction. Thus we define latent shares for the cases where purchases are observed as follows:

$$
s_{i h}^{*}=s_{i h}\left(1-\sum_{i \notin C} s_{i h}^{*}\right) \forall i \in C
$$

Pudney (1989) notes that implementing Wales and Woodland (1983) using maximum likelihood in systems with more than 3 commodities is computationally very expensive. This is because of the complex inter-dependencies which exist between the end points of the integrals in the likelihood function. The data augmentation algorithm which we employ does not entail any integration and it is therefore much less costly to implement Wales and Woodland (1983) using our method.

The AIDS is then expressed in terms of the latent shares as follows:

$$
\mathbf{s}^{*}=\mathbf{X}_{1} \mathbf{\Lambda}+\mathbf{v}
$$

where:

$$
\begin{gathered}
\mathbf{X}_{1}=\mathbf{I}_{m} \otimes \mathbf{x}_{1}, \\
\mathbf{x}_{1}=\mathbf{x}_{11}, \ldots, \mathbf{x}_{1 H}, \\
\mathbf{x}_{1 h}=\left(1, \ln p_{1, h}, \mathrm{~L}, \ln p_{m+1, h}, \ln \left(\frac{e_{h}}{P_{h}}\right), D_{h}^{\prime}\right)^{\prime},
\end{gathered}
$$




$$
\begin{gathered}
\mathbf{s}^{*}=\left(s_{1,1}^{*}, \mathrm{~L}, s_{1, H}^{*}, s_{2,1}^{*}, \ldots, s_{2, H}^{*}, \ldots, s_{m, 1}^{*}, \ldots s_{m, H}^{*}\right)^{\prime}, \\
\boldsymbol{\Lambda}=\alpha_{1}, \gamma_{11}, \ldots \gamma_{1, m+1}, \omega_{1}, \psi_{1,}^{\prime} \ldots, \alpha_{m}, \gamma_{m 1}, \ldots \gamma_{m, m+1}, \omega_{m}, \psi_{m,}^{\prime}, \\
\mathbf{v}=\left(v_{1,1}, \mathrm{~L}, v_{1, H}, v_{2,1}, \ldots, v_{2, H}, \ldots, v_{m, 1}, \ldots v_{m, H}\right)^{\prime}
\end{gathered}
$$

$p_{j h}$ is the price of the $j^{t h}$ good to the $h^{\text {th }}$ household $e_{t}$ is total expenditure, $P_{h}=\prod_{j} p_{j h}^{s_{j h}}$ is the

Stone's price index and $D_{h}$ is a vector of variables that describes demographic features of the $h^{\text {th }}$ household.

The underlying theory requires that the model satisfies symmetry

$$
\gamma_{i j}=\gamma_{j i} \text { for all } i, j
$$

homogeneity

$$
\sum_{j} \gamma_{i j}=0 \text { for all } j
$$

and concavity. Concavity implies that the Slutsky matrix (M) which has the elements:

$$
\begin{gathered}
M_{i j}=\gamma_{i j}+\omega_{i} \omega_{j} \ln \left(\frac{e}{P}\right)-s_{i} \delta_{i j}+s_{i} s_{j} \\
\delta_{i i}=1, \delta_{i j}=0 \text { for } i \neq j
\end{gathered}
$$

is negative semi-definite. The restrictions required for symmetry and homogeneity can be written in the form

$$
\mathrm{R} \Lambda^{*}=\mathbf{0}
$$

where $\mathbf{R}$ is an $r \times m(m+2)$ matrix defining the restrictions and $\Lambda^{*}$ is the restricted $\Lambda$. In order to impose these restrictions we reparameterise the model as follows. First define the $(\mathrm{km}-r) \times k m$ orthonormal matrix $\mathbf{R}_{\perp}$ such that:

$$
\begin{gathered}
\mathbf{R R}_{\perp}^{\prime}=0 \\
\mathbf{R}_{\perp} \mathbf{R}_{\perp}^{\prime}=\mathbf{I} .
\end{gathered}
$$

The restricted $\Lambda$ can be expressed as:

$$
\Lambda^{*}=\mathbf{R}_{\perp}^{\prime} X^{\prime}
$$

where $\not^{\circ}$ is a $(\mathrm{km}-\mathrm{r}) \times 1$ vector of distinct parameters. By substituting equation (20) into equation (6) the restricted model can be written:

$$
\begin{gathered}
\mathbf{s}^{*}=\mathbf{X}_{1} \mathbf{R}_{\perp}^{\prime} \not^{o}+\mathbf{v} \\
\mathbf{s}^{*}=\mathbf{W} \not^{o_{+}} \mathbf{v}
\end{gathered}
$$

where:

$$
\mathbf{W}=\mathbf{X}_{1} \mathbf{R}_{\perp}^{\prime} .
$$

Equation (22) is the basis for estimation and the restricted parameter vector is recovered using equation (20).

To complete the IPM, the demand equations in (6) are combined with $m$ probit equations to give the complete model: 


$$
\begin{aligned}
& \mathbf{s}^{*}=\mathbf{W} \AA^{0}+\mathbf{v} \\
& \mathbf{y}^{*}=\mathbf{X}_{2} \Gamma+\mathbf{u}
\end{aligned}
$$

where $\mathbf{y}^{*}$ is an $m H \times 1$ vector of latent variables structured in the same way as $\mathbf{s}^{*}$ (see equation 10) and based on the binary variable $y_{i h}$ defined in equation (1):

$$
\begin{gathered}
y_{i h}^{*}\left\{\begin{array}{lll}
> & y_{i h}=1 \\
\leq & 0 & y_{i h}=0
\end{array}\right. \\
\mathbf{e}=\left(\begin{array}{l}
\mathbf{v} \\
\mathbf{u}
\end{array}\right) \sim N \quad 0, \boldsymbol{\Sigma} \otimes \mathbf{I}_{H},
\end{gathered}
$$

and

$$
\begin{gathered}
\mathbf{X}_{2}=\mathbf{I}_{m} \otimes \mathbf{x}_{2} \\
\mathbf{x}_{2}=\mathbf{x}_{21} \ldots, \mathbf{x}_{2 H},
\end{gathered}
$$

is a matrix of variables that describe household specific characteristics which are assumed to determine the probability of the household making a purchase in a given time period. ${ }^{3}$ In our application we assume that all households are identical in this respect and stocks are exhausted in a purely random manner. $\mathbf{x}_{2}$ is therefore a vector of constants. Since the dependent variables in the probit equations (25) are unobserved, data augmentation is also used in their estimation. With the introduction of the probit equations the probability that is necessary for the computation of the latent shares in equation (2) can be obtained as:

$$
\Phi_{i h}=p \quad y_{i h}=1=p \quad y_{i h}^{*}>0=p \quad u_{i h}>-\mathbf{x}_{2 h} \boldsymbol{\Gamma}_{\mathbf{i}}=\Phi \quad \mathbf{x}_{2 h} \boldsymbol{\Gamma}_{\mathbf{i}}
$$

where $\boldsymbol{\Gamma}_{\mathbf{i}}$ is the sub-vector of $\boldsymbol{\Gamma}$ corresponding to the $i^{t h}$ probit equation.

\section{Bayesian Inference}

We apply Bayesian inference to the parameters of the model by sampling from the posterior distribution of the parameters in the model and presenting the summary statistics of this sample. The Gibbs sampler (see Casella and George, 1992) allows one to sample from a marginal distribution by using the conditional distributions of the parameters. In most applications parameters are grouped into blocks and the conditional distributions for these blocks are used as the basis for the sampler. If the dependent variables in equations (24) and (25) were observable, the full system comprising both sets of equation could be treated as a set of seemingly unrelated equations (SUR) and estimation using a Gibbs sampler would be straightforward. Writing the complete system in equations (24) and (25) as:

$$
\mathbf{z}^{*}=\mathbf{X} \beta+\mathbf{e}
$$

where:

$$
\mathbf{z}^{*}=\mathbf{s}^{*^{\prime}}, \mathbf{y}^{*^{\prime}}, \mathbf{X}=\left(\begin{array}{cc}
\mathbf{W} & 0 \\
0 & \mathbf{X}_{2}
\end{array}\right), \beta=\ell^{b}, \Gamma^{\prime}
$$

Assuming a diffuse prior (Zellner,1971, p 241):

\footnotetext{
${ }^{3}$ The stochastic specification of our IPM differs slightly from that of Blundell and Meghir (1987) who explicitly allow for errors in both the decision about whether to consume and the decision about how much to consume. We implicitly assume that both sources of error are represented by the residuals in $\mathrm{v}$.
} 


$$
p \boldsymbol{\beta}, \boldsymbol{\Sigma}^{-1}=p \boldsymbol{\beta} p \boldsymbol{\Sigma}^{-1} \propto\left|\boldsymbol{\Sigma}^{-1}\right|^{-\left(\frac{m+1}{2}\right)}
$$

the conditional posterior distributions for the two blocks of parameters $\boldsymbol{\beta}$ and $\boldsymbol{\Sigma}$ are:

$$
\begin{gathered}
p \boldsymbol{\beta} \mid \mathbf{z}, \mathbf{X}, \Sigma \sim M V N \quad \Sigma^{-1} \otimes \mathbf{X}^{\prime} \mathbf{X}^{-1} \Sigma^{-1} \otimes \mathbf{X}^{\prime} \quad \mathbf{z}^{*}, \Sigma^{-1} \otimes \mathbf{X}^{\prime} \mathbf{X} \\
p \Sigma \mid \mathbf{y}, \mathbf{X}, \boldsymbol{\beta} \sim I W \quad \mathbf{\varepsilon}, \mathbf{H}
\end{gathered}
$$

where:

$$
\boldsymbol{\theta}=\left(\begin{array}{rrrrrr}
v_{1,1} & \ldots & v_{m, 1} & u_{1,1} & \ldots & u_{m, 1} \\
\mathbf{M} & & \mathbf{M} & \mathbf{M} & & \mathbf{M} \\
v_{1, H} & \ldots & v_{m, H} & u_{1, H} & \ldots & u_{m, H}
\end{array}\right)
$$

As has been stated above, the theoretically derived property of concavity requires that the Slutsky matrix of the cost function (see equation 15) to be negative semi-definite. This is incorporated in the estimation by introducing an informative prior in the form of an indicator function which takes the value of one when the parameter vector $\boldsymbol{\beta}$ leads to a negative semi-definite Slutsky matrix and zero otherwise. In practice this results in an accept/reject step in the algorithm in which only those draws on the distribution in equation (34) which satisfy this restriction are retained in the sample that is used for inference.

We have noted above that some elements of $\mathbf{z}^{*}$ are not observed however. In order to complete the algorithm we therefore employ data augmentation. Data augmentation was introduced by Tanner and Wong (1987) as a method for conducting inference on the full posterior in the presence of latent data. Albert and Chib (1993) show how data augmentation can be accomplished using the Gibbs sampler. They show that where the conditional distributions of the latent data can be obtained, these data can be treated as another block of unknowns in the algorithm. In section 2 we argued that there were three types of latency in our model. The first type of latency is common to all limited dependent variable models and is referred to as missing data. In our model we have two types of missing data. In the share equations, where no purchase is made the shares are missing. In the probit equations the continuous variable $y_{i h}^{*}$ which underlies the observed binary variable is missing. The remaining two sources of latency apply to the observations where a purchase is made. In these cases latency exists first because the observed purchases do not correspond to actual consumption and second because the adding up restriction will not hold once consumption of the commodities where no purchase is made is accounted for. In all three cases the conditional distributions of the latent data are used in the algorithm to simulate values.

Let us turn to the derivation of these distributions. First consider the conditionals for the missing data. Because the observations for individual households are assumed to be independent we can make the latent draws household by household. In order to introduce the conditional distributions therefore we define $\mathbf{z}_{h}^{*}$ and $\hat{\mathbf{z}}_{h}^{*}$ to include only the elements of $\mathbf{z}^{*}$ and $\mathbb{\mathbf { z }}^{*}=\mathbf{X} \boldsymbol{\beta}$ respectively corresponding to the $h^{\text {th }}$ household. It is also more convenient to draw the latent variables commodity by commodity. Therefore, defining the precision matrix $\boldsymbol{\Omega}=\boldsymbol{\Sigma}^{-1}$, the conditional mean $\mu_{h t}$ and variance $V_{i}$ of the individual elements of $\mathbf{z}^{*}$ are (Geweke, 2005, Theorem 5.3.1):

$$
\begin{gathered}
\mu_{i h}=\hat{z}_{i h}^{*}+\Sigma_{i} \Sigma_{-i}^{-1} \mathbf{z}_{-i, h}^{*}-\hat{\mathbf{z}}_{-i, h}^{*}=\hat{z}_{i h}^{*}-\Omega_{i i}^{-1} \boldsymbol{\Omega}_{-i} \mathbf{z}_{-i, h}^{*}-\hat{\mathbf{z}}_{-i, h}^{*} \\
V_{i}=\Sigma_{i i}-\Sigma_{\mathbf{i}} \Sigma_{-\mathbf{i}}^{-1} \Sigma_{-\mathbf{i}}^{\prime}=\boldsymbol{\Omega}_{-\mathbf{i}}^{-1}
\end{gathered}
$$

where $\Sigma_{\mathrm{ii}}$ is the $i^{\text {th }}$ on-diagonal element of $\boldsymbol{\Sigma}, \boldsymbol{\Sigma}_{i}$ is the $i^{\text {th }}$ row of $\boldsymbol{\Sigma}$ excluding $\Sigma_{i i}$, and $\boldsymbol{\Sigma}_{-i}$ is the 
matrix within $\boldsymbol{\Sigma}$ excluding both the $i^{\text {th }}$ column and $i^{\text {th }}$ row. $\Omega$ ii and $\boldsymbol{\Omega}_{i}$ are similarly defined. $\hat{z}_{i h}$ is the fitted value of $z_{i h}$ for the $h^{\text {th }}$ household and $\hat{\mathbf{y}}_{-i, h}$ and $\mathbf{y}_{-i, h}$ are vectors within $\hat{\mathbf{y}}_{h}$ and $\mathbf{y}_{h}$ respectively, with their $i^{t h}$ elements removed. The conditional distributions for the missing data in the probit equations are:

$$
\begin{gathered}
y_{i h}=0 \text { for } y_{i h}^{*} \mid \mathbf{y}_{-i, h}^{*}, \beta, \mathbf{X}, \Sigma \sim \mathbf{N} \mu_{i h}, V_{i} I_{[-\infty, 0]} \forall i, h \\
y_{i h}=1 \text { for } y_{i h}^{*} \mid \mathbf{y}_{-i, h}^{*}, \beta, \mathbf{X}, \Sigma \sim \mathbf{N} \mu_{i h}, V_{i} I_{[0, \infty]} \forall i, h
\end{gathered}
$$

and in the share equations:

$$
s_{i h}=0 \text { for } s_{i h}^{*} \mid \mathbf{y}_{-i, h}^{*}, \Theta, \mathbf{X}, \Sigma \sim \mathbf{N} \mu_{i h}, V_{i} I_{[0,1]} \forall i \notin C, h
$$

where $I_{[-\infty, 0]}$ is an indicator variable that is one if $y_{i t} \in-\infty, 0$ and zero otherwise and $I_{[0,1]}$ is similarly defined on the interval from zero to one.

For the remaining two types of latency, in observations where a purchase is made, the latent data are a linear transformation of the observed data. This data can therefore be simulated by applying the transformations in equations (2) and (5) sequentially to the observed data. It can be seen that because our method simulates the latent data and estimates the model directly using these data it greatly simplifies the Wales and Woodland (1983, p270) approach to ensuring that adding up is satisfied by the latent shares in comparison with maximum likelihood.

The remaining issue we shall discuss is the identification of the probit equations. To achieve this it is necessary to restrict the covariance matrix:

$$
\boldsymbol{\Sigma}=\left(\begin{array}{ll}
\boldsymbol{\Sigma}_{v v} & \boldsymbol{\Sigma}_{v u} \\
\boldsymbol{\Sigma}_{u v} & \boldsymbol{\Sigma}_{u u}
\end{array}\right) .
$$

and we impose the restriction that $\Sigma_{\mathrm{uu}}=\mathrm{I}$. Instead of using equation (35) as the basis for making draws on $\Sigma$, we obtain the conditional posterior distributions for the sub-matrices within $\Sigma$ as follows. Define the following $H \times m$ matrices:

$$
\begin{aligned}
& \boldsymbol{\theta}=\left(\begin{array}{cccc}
v_{11} & v_{21} & \mathrm{~L} & v_{m 1} \\
\mathrm{M} & \mathrm{M} & & \mathrm{M} \\
v_{1 H} & v_{2 H} & \mathrm{~L} & v_{m H}
\end{array}\right) \\
& \mathbf{v} \mathbf{c}=\left(\begin{array}{rrrr}
u_{11} & u_{21} & \mathrm{~L} & u_{m 1} \\
\mathrm{M} & \mathrm{M} & & \mathrm{M} \\
u_{1 H} & u_{2 H} & \mathrm{~L} & u_{m H}
\end{array}\right)
\end{aligned}
$$

From the properties of the multivariate normal the conditional mean and variance are:

$$
\begin{gathered}
E(\mathbf{v} \mid \mathbf{u})=\boldsymbol{\Sigma}_{v u} \boldsymbol{\Sigma}_{u u}^{-1} \mathbf{u} \\
E \mathbf{v}^{\prime} \mathbf{v} \mid \mathbf{u}=\boldsymbol{\Sigma}_{\varepsilon}=\boldsymbol{\Sigma}_{v v}-\boldsymbol{\Sigma}_{v u} \boldsymbol{\Sigma}_{u u}^{-1} \boldsymbol{\Sigma}_{v u}
\end{gathered}
$$

where 45 is a regression of $\mathrm{v}$ on $\mathrm{u}$. Under the assumption that $\boldsymbol{\Sigma}_{u u}=\mathbf{I}$ we can therefore parameterise the covariance matrix as:

$$
\boldsymbol{\Sigma}=\left(\begin{array}{ll}
\boldsymbol{\Sigma}_{v v} & \boldsymbol{\Sigma}_{v u} \\
\boldsymbol{\Sigma}_{u v} & \boldsymbol{\Sigma}_{u u}
\end{array}\right)=\left(\begin{array}{cc}
\boldsymbol{\Sigma}_{\varepsilon}+\boldsymbol{\rho} \boldsymbol{\rho} & \boldsymbol{\rho} \\
\boldsymbol{\rho}^{\prime} & \mathbf{I}
\end{array}\right) .
$$

where $\boldsymbol{\rho}=\Sigma_{\mathrm{vu}}$. Recognising from equations (45) and (46), with the assumption $\boldsymbol{\Sigma}_{u u}=\mathbf{I}$, that $\boldsymbol{\rho}$ and 
$\Sigma_{\varepsilon}$ are the coefficient vector and covariance matrix of the error term $\boldsymbol{\varepsilon}$ respectively in the following seemingly unrelated regression:

$$
\mathbf{v}=\boldsymbol{\rho} \mathbf{u}+\boldsymbol{\varepsilon}
$$

with $\varepsilon \sim N 0, \Sigma_{\varepsilon}$ and assuming a diffuse prior, we use the following conditional posteriors:

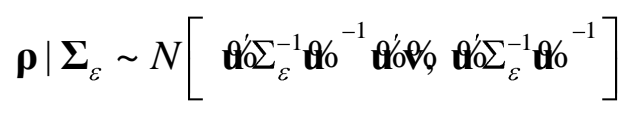

and

$$
\boldsymbol{\Sigma}_{\varepsilon} \mid \boldsymbol{\delta} \sim I W\left(\boldsymbol{\varepsilon}^{\prime} \boldsymbol{\varepsilon}, \mathbf{H}\right)
$$

together with the relations in equation (47) as the basis for sampling the restricted covariance matrix.

The estimation algorithm can then be stated as:

1. Assume starting values for $\mathbf{z}^{*}$ and $\boldsymbol{\Sigma}$.

2. Use the most recently drawn values of $\mathbf{z}^{*}$ from steps 4 and 5 and $\boldsymbol{\Sigma}$ from step 6 (or those assumed in step 1 if this is the first pass), draw the parameter vector $\boldsymbol{\beta}$ from the normal distribution in equation (34).

3. Use the appropriate elements of the $\boldsymbol{\beta}$ draw to compute the Slutsky matrix using equation (15) and check to see whether it is negative semi-definite. If it is, add the draw to the sample. If it is not, revert to the previous draw of $\boldsymbol{\beta}$.

4. Using the parameter vector drawn in step 2, compute $\mathbb{Z}^{*}=\mathbf{X} \boldsymbol{\beta}$. Using the appropriate elements in $\mathbf{Z}^{*}$ and $\boldsymbol{\Sigma}$ from step 6 (or that assumed in step 1 if this is the first pass), compute the mean and variance of the conditional distributions using equations (37) and (38). Use these in the truncated normal distributions in equations (39) and (40) to draw the latent data for the probit equations.

5. Obtain the latent data for the share equations:

a) Where the share is censored use the appropriate elements in $\mathbf{Z}^{*}$ from step 4 and $\mathbf{\Sigma}$ from step 6 (or that assumed in step 1 if this is the first pass) to compute the mean and variance of the conditional distribution using equations (37) and (38). Use these to make a draw on the distribution in equation (41).

b) Where a purchase is observed compute the probability of a purchase using equation (30) and the latent shares using equations (4) and (5).

6. Using $\boldsymbol{\beta}$ from step 2 and $\mathbf{z}^{*}$ from steps 4 and 5, draw the variance-covariance matrix $\boldsymbol{\Sigma}$ :

a) Draw $\boldsymbol{\rho}$ from the normal distribution in equation (49).

b) Draw $\Sigma_{\varepsilon}$ from the inverse Wishart distribution in equation (50).

c) Construct the complete matrix using equation (47).

7. Return to step 2.

\section{Data and aggregation}

We use the United Kingdom (UK) government's Expenditure and Food Survey (EFS) for 2003-4. Participating households voluntarily record food purchases for consumption at home for a two week period using a food diary. The sample is based on 7,014 households in 672 postcode sectors stratified by Government Office Region, socioeconomic group and car ownership. It is carried out throughout the UK and throughout the year in order to capture seasonal variations.

We estimate three models which are based on subsets of foods aggregated in such a way to be of particular interest from the perspective of dietary health policy. The three groups are respectively: 
the 'balance of good health', fish and fruit and vegetables. In all cases observations are excluded where none of the food groups in the model are consumed. This leaves 7,014, 4,914 and 6,800 observations for the balance of good health, fish and fruit and vegetables models respectively. The balance of good health model comprises the following groups: milk and dairy; meat, fish and alternatives; bread, cereals and potatoes; fats and sugar and fruit and vegetables. These are chosen because they correspond to groups used by the UK Food Standards Agency (FSA) in recommendations regarding what represents a balanced diet. In this model, levels of censoring vary from $0.54 \%$ for the cereals and potatoes group to $3.36 \%$ for fruit and vegetables. In the Fish model we estimate demand equations for: white fish; salmon; blue fish; shellfish and other fish. This model was chosen because oily fish have been shown to have beneficial health impacts and there are therefore concerns about low levels of consumption in some groups. Here the levels of censoring range from $22.62 \%$ for other fish to $85.70 \%$ for shellfish. Finally, the fruit and vegetables model comprises demand equations for: fresh fruit and vegetables; frozen fruit and vegetables; tinned fruit and vegetables; prepared fruit and vegetables and fruit and vegetable based ready meals. The levels of censoring in this model range from 3.6\% for fresh fruit and vegetables to $70.69 \%$ for frozen fruit and vegetables. This aggregation was chosen because of the objective to increase consumption of fresh fruit and vegetables. ${ }^{4}$ Prices are not available in the EFS and we therefore follow what has become common practice (Yen et al., 2003, Yen and Lin, 2006) in using unit values to represent household prices and by imputing the missing prices for censored observations as regional averages. ${ }^{5}$ These are computed using the same data on households for which purchases are observed within the appropriate region. We recognise that alternatives to this approach exist, for example Deaton (1988) and Deaton (1990) address the problems associated with using unit values as opposed to prices and as Yen et al. (2003) note, Rubin (1996) offers a more robust methods for imputation. We argue however that these methods are beyond the scope of this paper.

[Table 1 about here.]

Demographic characteristics are included in the demand system by augmenting each of the share equations with a vector $\mathbf{h}_{\mathrm{t}}$ of dummy variables to represent the characteristics listed in table 1 .

\section{Results}

Tables 2 to 4 show the price elasticities calculated using the following formula:

$$
\grave{\mathrm{O}}_{i j}=-\delta_{i j}+\frac{\bar{\gamma}_{i j}}{\bar{s}_{i}}-\bar{\omega}_{i} \frac{\bar{s}_{j}}{\bar{s}_{i}},
$$

where $\bar{\gamma}_{i j}$ and $\bar{\omega}_{i}$ are the means of the draws in the MCMC sample corresponding to the parameters defined in equation 6. $s_{i}$ is the mean value of the $i^{\text {th }}$ share across all observations in the data set and:

$$
\left\{\begin{array}{l}
\delta_{i i}=1 \\
\delta_{i j}=0 \quad i \neq j
\end{array} .\right.
$$

The expenditure elasticities in tables 2 to 4 are calculated as:

$$
\grave{\mathrm{o}}_{i}=1+\frac{\bar{\omega}_{i}}{\bar{s}_{i}}
$$

We also report the highest posterior density intervals based on the 2.5 and 97.5 centiles in the MCMC sample and these show that a very high proportion of the estimated elasticities are significant in the sense that the interval does not span zero.

\footnotetext{
${ }^{4}$ Full details of the foods included in each of these models are available on request.

${ }^{5}$ Note also that, following (Alston et al 2001), our elasticity estimate may not be invariant to differential scaling of the variables in the model.
} 
The elasticities for the balance of good health model that are reported in table 2

[Table 2 about here.]

show that all of the foods are own price inelastic with milk and dairy the least responsive and meat and fish the most responsive. All of the significant cross price effects show the goods to be complementary emphasising the importance of the income effect in determining cross price responsiveness. This is a pattern that is repeated in the other two models albeit to a lesser extent and it raises questions about the use of differential pricing through taxation and subsidies in order to induce substitution from healthy to unhealthy foods. For example there is a comparatively strong complementary relationship between the price of fruit and vegetables and the quantity of cereals, bread and potatoes. Thus a subsidy on fruit and vegetables may be expected to have an undesirable impact on the quantity consumed of high calorie cereals, bread and potatoes. The effects may not all be undesirable: there is also a comparatively strong complementarity between the price of fats and sugars, a group which includes butter, jams, biscuits cakes and sweets, and meats, fish etc. This suggests that a "fat tax" may also have a beneficial impact in reducing consumption of red meats. The large number of complementary relationship may be unexpected. We do not report the Hicksian elasticities in the interests of brevity but examination of these reveals that, without exception, the compensated cross price effects are all substitutes. Thus we attribute the preponderance of complementarity to the expenditure effects.

The expenditure elasticities show the impacts on demand for the individual goods of changes in expenditure on all foods within the system in question. These indicate therefore the relative effects of changes in income on the different food groups although we would expect the magnitude of the true income elasticities of demand to be smaller than these expenditure elasticities. ${ }^{6}$ Milk and dairy, fats and sugar and cereals bread and potatoes are expenditure inelastic whilst meat, fish etc and fruit and vegetables are expenditure elastic. This implies that households with higher levels of expenditure will consume a relatively higher proportion of meat and of fruit and vegetables.

[Table 3 about here.]

In table 3 we see that all fish except for shellfish are own price inelastic. The table also shows that all fish except shellfish are income inelastic. Blue fish and salmon are unusual in so far as they run counter to the general pattern of complementarity that is observed in the majority of cases. The fact that these two are substitutes is perhaps not surprising given that they are both oily fish. The expenditure elasticities suggest that there is likely to be a higher proportion of oily fish in comparison with white fish in the diets of high income households.

[Table 4 about here.]

Table 4 also shows that all of the groups within this category are price inelastic. The most notable feature of these results from the dietary health perspective is that the only expenditure elastic group is fresh fruit and vegetables. Thus not only do higher income households spend proportionately more on fruit and vegetables as a whole (c.f. table 2) but within the fruit and vegetable category they spend proportionately more on the fresh products.

[Table 5 about here]

We turn now to the effects of the demographic variables on demand. We report results in table 5 for the food groups in the balance of good health model. We also indicate with an asterisk when the 95\% highest posterior density interval excludes zero as a measure of the significance of the results. The effects are estimated as the coefficients on dummy variables in the share equations and converted so that they measure the marginal effect on consumption over a two week period in natural units at the mean shares and prices across all households. All of the results show the effect

\footnotetext{
${ }^{6}$ The true income elasticity is the product of the expenditure elasticities which we report and the elasticity of food expenditure with respect to income. We expect the latter to be inelastic and as a result the true income elasticities for the food groups which we examine are expected to be smaller in magnitude than the results we report.
} 
relative to the reference group defined in table 1 . The values are based on the mean values of the parameters in the Gibbs sample. The results show that household composition has an impact across all of the food groupings. The presence of children in a household leads to a lower level of demand for fruit and vegetables and meat and increased demand for milk and dairy and cereals and potatoes. The demographic group has the most significant impacts on cereals and potato and on fruit and vegetable demand, where there is evidence that lower demographic groups substitute fruit and vegetables with cereals and potatoes. Demand for fats and sugar and for fruit and vegetables increases with age whilst demand for cereals and potatoes falls. Regional variation in the demand for fruit and vegetables is pronounced with the highest demand in London and the South East and the lowest in Scotland and Northern Ireland. Where the woman is responsible for food purchases, demand for fats and sugar and for fruit and vegetables is increased whilst that for meat is reduced. Ethnicity has the most significant impact on demand for fruit and vegetables with increased demand amongst all groups in comparison with the reference white households.

\section{Conclusions}

We have demonstrated how the infrequency of purchase model can be estimated for a system of equations using Monte Carlo Markov chain methods. The method has been illustrated by estimating a model which is designed to disentangle the impacts of economic factors from preference heterogeneity resulting from differing demographic conditions in influencing the healthiness of diets in England and Wales.

Our results imply that households which have a higher level of expenditure will tend to consume proportionately more meat and more fresh fruit and vegetables. Households in London and the South East have higher levels of fruit and vegetable consumption whilst it is reduced by the presence of children. Households employed in the professional or managerial sectors also have higher levels of fruit and vegetable consumption. Age has an influence on the consumption of fats and sugars with consumption declining amongst older households. Age also has an impact on the types of fruit and vegetables consumed with younger households preferring more ready meals and prepared fruit and vegetables.

Overall the results emphasise the role played by low incomes and socio-economic circumstances in poor dietary choices. Taking the example of fruit and vegetables we have shown that, comparing an unemployed individual with an otherwise identical individual living in a household of two, we would expect the former to consume over $3 \mathrm{~kg}$ less fruit. Similarly, for two identical households a difference in income of $10 \%$ can be expected to lead to a difference in demand for fruit and vegetables of around $500 \mathrm{~g}$. The other aspect of the results which is especially important in a policy context is the effect of the presence of children in households. Remaining with the example of fruit and vegetables, comparing a two person household with children with one without them would reveal an average level of fruit and vegetable demand which is almost $2 \mathrm{~kg}$ higher in the childless family. These patterns are repeated across all of the dietary components that we have analysed and have important implications for policy makers. There are clear distributional implications for dietary health that arise from these patterns of consumption and also for the health of children. They suggest that targeted interventions are necessary in order to reduce the incidence of diet related health problems in the future.

\section{Acknowledgements}

We are grateful to two anonymous referees for helpful suggestions in improving this paper and to the Food Standards Agency for providing financial support in the early stages of this research. Andreas Anastassiou worked on early versions of the code used in estimating the models.

\section{References}

J.H. Albert and S. Chib. (1993) Bayesian Analysis of Binary and Polychotomous Response Data. 
Journal of the American Statistical Association 88:669-679.

Alston J.M., J.A. Chalfant, and N.E. Piggott. (2001) Incorporating Demand Shifters in the Almost Ideal Demand System. Economics Letters 70:73-78.

Amemiya T. (1974) Multivariate Regression and Simultaneous Equation Models when Dependent Variables are Truncated Normal. Econometrica 42:999 - 1012.

Blundell R. and C. Meghir. (1987) Bivariate Alternatives to the Tobit-model. Journal of Econometrics 34:179 - 200.

Casella G. and E. I. George. (1992) Explaining the Gibbs Sampler. The American Statistician 46:167-174.

Cragg J.G. (1971) Some Statistical Models for Limited Dependent Variables with Application to Demand for Durable Goods. Econometrica 39:829 - 844.

Deaton A. (1988) Quality, Quantity and Spatial Variation of Price. American Economic Review 78:418-430.

Deaton A. (1990) Price elasticities from survey data: Extensions and Indonesian results. Journal of Econometrics 44:281-309.

Dowler E. (2003) Food and poverty. Development Policy Review 21:569-580.

Drewnowski A. (2004) Obesity and the food environment - dietary energy density and diet costs. American Journal of Preventive Medicine 27:154 - 162.

Geweke J. (2005) Contemporary Bayesian Econometrics and Statistics. New Jersey, US: Wiley.

Heckman J.J. (1979) Sample selection bias as a specification error. Econometrica 47:153-161.

Heien D. and C.R. Wessells. (1990) Demand systems estimation with microdata - a censored regression approach. Journal of Business and Economic Statistics 8:365 - 371.

Phaneuf D.J. C.L. Kling, and J.A. Herriges. (2000) Estimation and welfare calculations in a generalized corner solution model with an application to recreation demand. Review of Economics and Statistics 82:83-92.

Pudney S. (1989) Modelling Individual Choice: The Econometrics of Corners Kinks and Holes. Oxford, UK: Blackwell.

Rubin D.B. (1996) Multiple imputation after 18+ years. Journal of the American Statistical Association 91:473-89.

Shonkwiler J.S. and S.T. Yen. (1999) Two-step estimation of a censored system of equations. American Journal of Agricultural Economics 81:972 - 982.

Stewart H. and S.T. Yen. (2004) Changing household characteristics and the away-from-home food market: a censored equation system approach. Food Policy 29:643 - 658.

Tanner M.A. and W.H. Wong. (1987) The calculation of the posterior distribution by data augmentation (with discussion). Journal of the American Statistical Association 82:528-550.

Tobin J. (1958) Estimation of relationships for limited dependent variables. Econometrica 26:2436.

Wales T.J. and A.D. Woodland. (1983) Estimation of consumer demand systems with binding non-negativity constraints. Journal of Econometrics 21:263 - 285.

Yen S.T. (2005) A multivariate sample-selection model: Estimating cigarette and alcohol demands with zero observations. American Journal of Agricultural Economics 87:453 - 466.

Yen S.T. and B.H. Lin. (2006) A sample selection approach to censored demand systems. American Journal of Agricultural Economics 88:742 - 749. 
Yen S.T. B.H. Lin, and D.M. Smallwood. (2003) Quasi- and simulated-likelihood approaches to censored demand systems: Food consumption by food stamp recipients in the United States. American Journal of Agricultural Economics 85:458 - 478.

Zellner A. (1971) An Introduction to Bayesian Analysis in Econometrics. New York, US: Wiley (reprinted 1996 as "Wiley Classics" edition). 


\section{List of Tables}

1 Demographic Variables Included in the Share Equations

2 Elasticities of Demand for the Balance of Good Health Model

3 Elasticities of Demand for the Fish Model

4 Elasticities of Demand for the Fruit and Vegetables Model

5 Effects of Socio-demographic Variables on Food Demand 
Table 1: Demographic Variables Included in the Share Equations

\begin{tabular}{|c|c|}
\hline $\begin{array}{l}\text { Household } \\
\text { Composition }\end{array}$ & $\begin{array}{l}\text { Adults only* - Single parents - Family with children - Family with children \& } \\
\text { more than } 2 \text { adults - Family without children \& more than } 2 \text { adults }\end{array}$ \\
\hline $\begin{array}{l}\text { Socio-economic } \\
\text { Group }^{\text {a }}\end{array}$ & $\begin{array}{l}\text { High managerial* }{ }^{*} \text { - Low managerial - Workers-technical - Never } \\
\text { work/unemployed - Students - Other }\end{array}$ \\
\hline $\operatorname{Age}^{\mathrm{a}}$ & $(<30)^{*}-(30-44)-(45-60)-(\geq 60)$ \\
\hline $\mathrm{GOR}^{\mathrm{b}}$ & $\begin{array}{l}\text { North East - North West \& Merseyside - Yorks \& Humber - East Midlands - } \\
\text { West Midlands - Eastern - London* - South East - South West - Wales - } \\
\text { Scotland - Northern Ireland }\end{array}$ \\
\hline Ethnic Origin ${ }^{\mathrm{a}}$ & White* - Mixed race - Asian - Black - Other \\
\hline Gender $^{\mathrm{a}}$ & Male* - Female \\
\hline
\end{tabular}

${ }^{\text {a }}$ Relating to the household reference person (HRP)

${ }^{\mathrm{b}}$ Government Office Region

* indicates the omitted dummy variable in each category, thereby defining the reference demographic group for interpreting results 
Table 2: Elasticities of Demand for the Balance of Good Health Model

Density

intervals
Price

Expenditure

Quantity

Dairy Meat Fats Cereals F and V

$\begin{array}{ccccccc}\text { Milk and Dairy Point estimate } & -0.202 & -0.089 & -0.093 & -0.172 & -0.156 & 0.718 \\ 2.50 \% & -0.315 & -0.173 & -0.155 & -0.246 & -0.218 & 0.692 \\ 97.50 \% & -0.136 & -0.001 & -0.036 & -0.095 & -0.092 & 0.742\end{array}$

Meat, Fish etc. Point estimate

$\begin{array}{lllll}-0.092 & -0.859 & -0.105 & -0.093 & -0.018\end{array}$

1.163

$2.50 \%$

$-0.123$

$-0.918-0.137$

$-0.13 \quad-0.05$

1.147

$97.50 \%$

$-0.059$

$\begin{array}{llll}-0.8 & -0.074 & -0.056 & 0.013\end{array}$

1.18

Fats

$\begin{array}{ccccccc}\text { Point estimate } & -0.108 & -0.161 & -0.525 & -0.11 & -0.026 & 0.93 \\ 2.50 \% & -0.161 & -0.235 & -0.597 & -0.171 & -0.081 & 0.906 \\ 97.50 \% & -0.059 & -0.101 & -0.456 & -0.05 & 0.029 & 0.955\end{array}$

Bread, Cereals,

Potatoes

$\begin{array}{ccccccc}\text { Point estimate } & -0.136 & -0.073 & -0.08 & -0.524 & -0.106 & 0.92 \\ 2.50 \% & -0.183 & -0.135 & -0.125 & -0.699 & -0.154 & 0.901 \\ 97.50 \% & -0.087 & -0.021 & -0.035 & -0.449 & -0.057 & 0.939\end{array}$

Fruit and

Vegetables

$\begin{array}{ccccccc}\text { Point estimate } & -0.155 & 0.009 & -0.038 & -0.144 & -0.71 & 1.038 \\ 2.50 \% & -0.199 & -0.053 & -0.085 & -0.2 & -0.776 & 1.014 \\ 97.50 \% & -0.109 & 0.061 & 0.008 & -0.089 & -0.645 & 1.057\end{array}$


Table 3: Elasticities of Demand for the Fish Model

\section{Density}

intervals
Price

Expenditure

Quantity

White Salmon Blue Shell Other

White

Point estimate

$\begin{array}{lll}-0.918 & 0.039 & 0.011\end{array}$

$\begin{array}{ll}0.152 & 0.06\end{array}$

0.873

$2.50 \%$

$-1.029$

$-0.061$

$-0.079$

$0.057 \quad-0.017$

0.825

$97.50 \%$

$-0.811$

0.155

0.1

0.252

0.128

0.924

Salmon

$\begin{array}{ccc}\text { Point estimate } & 0.016 & -0.79 \\ 2.50 \% & -0.115 & -0.915 \\ 97.50 \% & 0.146 & -0.663\end{array}$

$0.147 \quad 0.026$

$-0.194$

0.924

$97.50 \%$

0.022

$-0.101$

$-0.308$

0.828

0.284

$0.161-0.084$

0.992

Blue

Point estimate

$-0.007$

0.174

$-0.771 \quad-0.099$

$-0.06$

0.913

$2.50 \%$

$-0.145$

0.042

$-0.907$

$-0.259$

$-0.162$

0.818

$97.50 \%$

0.132

0.3

$\begin{array}{lll}-0.635 & 0.056 \quad 0.045\end{array}$

1.013

Shell

$\begin{array}{ccccccc}\text { Point estimate } & -0.075 & -0.168 & -0.265 & -1.041 & -0.324 & 1.321 \\ 2.50 \% & -0.212 & -0.302 & -0.407 & -1.224 & -0.434 & 1.194 \\ 97.50 \% & 0.074 & -0.027 & -0.13 & -0.857 & -0.2 & 1.439\end{array}$

Other

Point estimate

$-0.022$

$-0.163$

$-0.069$

$2.50 \%$

$-0.077$

$-0.163$

$-0.069$

$-0.053$

$-0.673$

0.993

$97.50 \%$

0.024

$-0.111$

$\begin{array}{lll}-0.115 & -0.094 & -0.739\end{array}$

0.96

1.031 
Table 4: Elasticities of Demand for the Fruit and Vegetables Model

Density

intervals
Price

Expenditure

Quantity

Ready Prepared Tinned Fresh Frozen

\begin{tabular}{|c|c|c|c|c|c|c|c|}
\hline \multirow[t]{3}{*}{ Ready } & Point estimate & -0.71 & -0.017 & 0.004 & -0.125 & 0.01 & 0.881 \\
\hline & $2.50 \%$ & -0.779 & -0.067 & -0.031 & -0.199 & 0.001 & 0.845 \\
\hline & $97.50 \%$ & -0.643 & 0.032 & 0.042 & -0.052 & 0.018 & 0.922 \\
\hline \multirow[t]{3}{*}{ Prepared } & Point estimate & 0 & -0.686 & 0.02 & -0.092 & 0.022 & 0.807 \\
\hline & $2.50 \%$ & -0.043 & -0.745 & -0.014 & -0.158 & 0.015 & 0.778 \\
\hline & $97.50 \%$ & 0.041 & -0.628 & 0.055 & -0.025 & 0.03 & 0.834 \\
\hline \multirow[t]{3}{*}{ Tinned } & Point estimate & 0 & 0.077 & -0.831 & 0.115 & 0.051 & 0.663 \\
\hline & $2.50 \%$ & -0.018 & 0 & -0.926 & 0.001 & 0.033 & 0.616 \\
\hline & $97.50 \%$ & 0.122 & 0.154 & -0.738 & 0.228 & 0.069 & 0.708 \\
\hline \multirow[t]{3}{*}{ Fresh } & Point estimate & 0.049 & -0.106 & -0.033 & -0.963 & -0.022 & 1.153 \\
\hline & $2.50 \%$ & -0.105 & -0.126 & -0.049 & -0.995 & -0.025 & 1.143 \\
\hline & $97.50 \%$ & -0.067 & -0.085 & -0.017 & -0.932 & -0.018 & 1.163 \\
\hline \multirow[t]{3}{*}{ Frozen } & Point estimate & 0.086 & 0.003 & 0.006 & 0.043 & -0.977 & 0.944 \\
\hline & $2.50 \%$ & -0.007 & -0.007 & -0.005 & 0.029 & -0.985 & 0.937 \\
\hline & $97.50 \%$ & 0.008 & -0.013 & 0.017 & 0.06 & -0.968 & 0.951 \\
\hline
\end{tabular}


Table 5: Effects of Socio-demographic Variables on Food Demand (asterisk indicates that the 95\% highest posterior density interval does not include zero).

\begin{tabular}{|c|c|c|c|c|c|c|}
\hline & & $\begin{array}{l}\text { Milk and } \\
\text { Dairy (ml) }\end{array}$ & Meat $(\mathrm{g})$ & $\begin{array}{l}\text { Fats and } \\
\text { Sugar }(g)\end{array}$ & $\begin{array}{c}\text { Cereals } \\
\text { and Potato } \\
(\mathrm{g})\end{array}$ & $\begin{array}{c}\text { Fruit and } \\
\text { Veg. (g) }\end{array}$ \\
\hline \multirow[t]{5}{*}{ Composition } & 1 or 2 adults only & - & - & - & - & - \\
\hline & Single parents & $1595.67 *$ & $-741.56^{*}$ & $614.84 *$ & $1732.67 *$ & $-1989.88 *$ \\
\hline & Children, 2 adults & $3011.05^{*}$ & $-1054.63 *$ & $751.38 *$ & $1399.89 *$ & $-1917.19 *$ \\
\hline & Children, $>2$ adults & $2327.19 *$ & $-909.17 *$ & 328.85 & $2644.41 *$ & $-2367.46^{*}$ \\
\hline & $>2$ adults, no children & $1416.25 *$ & $-498.80^{*}$ & 141.49 & $1429.81 *$ & $-1286.58 *$ \\
\hline \multirow{6}{*}{$\begin{array}{l}\text { Demographic } \\
\text { group }\end{array}$} & High managerial & - & - & - & - & - \\
\hline & Low managerial & -116.14 & 165.88 & 92.86 & $508.62 *$ & $-1146.25^{*}$ \\
\hline & Workers-technical & -512.24 & $404.40 *$ & 178.57 & $1437.68 *$ & $-2759.69 *$ \\
\hline & Never worked-unemp. & 441.17 & 178.02 & -6.59 & $2188.80 *$ & $-3144.59 *$ \\
\hline & Students & -1124.16 & 303.64 & -21.98 & $1463.92 *$ & $-1684.44 *$ \\
\hline & Other & -522.64 & 149.51 & $614.29 *$ & 310.21 & $-1690.08 *$ \\
\hline \multirow[t]{4}{*}{ Age } & Under 30 & - & - & - & - & - \\
\hline & Between 30 and 45 & $-836.40^{*}$ & $297.28 *$ & $382.42 *$ & $-1695.93 *$ & $710.63 *$ \\
\hline & Between 45 and 60 & -139.54 & $279.17 *$ & $781.33 *$ & $-3569.79 *$ & $1504.11 *$ \\
\hline & Over 60 & 365.76 & 259.52 & $1161.01 *$ & $-4642.67 *$ & $1606.11 *$ \\
\hline \multirow[t]{12}{*}{ Region } & North East & -602.38 & $415.96^{*}$ & 173.90 & $760.04 *$ & $-1997.21 *$ \\
\hline & NW \& Merseyside & 205.42 & $293.62 *$ & 243.68 & 178.99 & $-1683.88 *$ \\
\hline & Yorks. \& Humber & 40.74 & 113.67 & $345.33 *$ & 219.40 & $-1302.36^{*}$ \\
\hline & East Midlands & 656.99 & -100.38 & $467.04 *$ & -55.64 & $-1031.85^{*}$ \\
\hline & West Midlands & 186.35 & 229.65 & 242.31 & -74.53 & $-1209.94 *$ \\
\hline & Eastern & 296.42 & 44.12 & 196.71 & -266.12 & -439.57 \\
\hline & London & - & - & - & - & - \\
\hline & South East & $650.05 *$ & -125.42 & 175.28 & $-586.83 *$ & 214.71 \\
\hline & South West & 702.06 & -144.69 & $565.94 *$ & $-556.39 *$ & $-596.80 *$ \\
\hline & Wales & -292.96 & $446.21 *$ & 291.49 & -165.87 & $-1534.54 *$ \\
\hline & Scotland & -342.36 & 251.23 & $393.69 *$ & $783.14^{*}$ & $-2160.64 *$ \\
\hline & Northern Ireland & -200.22 & 1.93 & $314.29 *$ & $1787.26^{*}$ & $-2438.47 *$ \\
\hline \multirow[t]{2}{*}{ Sex } & Men & - & - & - & - & - \\
\hline & Women & -3.47 & $-208.65 *$ & $137.91 *$ & -224.65 & $570.87 *$ \\
\hline \multirow[t]{5}{*}{ Ethnicity } & White & - & - & - & - & - \\
\hline & Mixed & -1502.06 & -410.56 & $-954.68 *$ & 681.83 & $3403.82 *$ \\
\hline & Asian & -154.28 & $-1218.01 *$ & -90.11 & 735.90 & $3057.81 *$ \\
\hline & Black & $-2480.61 *$ & 326.18 & -292.59 & -689.71 & $1999.46^{*}$ \\
\hline & Other & $-3109.86^{*}$ & 387.44 & -134.89 & $-2767.76^{*}$ & $4137.00 *$ \\
\hline
\end{tabular}

